\title{
A Bio-Inspired QoS-Oriented Handover Model in Heterogeneous Wireless Networks
}

\author{
Daxin Tian, ${ }^{1,2}$ Jianshan Zhou, ${ }^{1,2}$ Honggang Qi, ${ }^{3}$ Yingrong Lu, ${ }^{1,2}$ Yunpeng Wang, \\ Jian Wang, ${ }^{1,2}$ and Anping $\mathrm{He}^{4}$ \\ ${ }^{1}$ Beijing Key Laboratory for Cooperative Vehicle Infrastructure Systems \& Safety Control, \\ School of Transportation Science and Engineering, Beihang University, Beijing 100191, China \\ 2 Jiangsu Province Collaborative Innovation Center of Modern Urban Traffic Technologies, Beijing 100191, China \\ ${ }^{3}$ School of Computer and Control Engineering, University of Chinese Academy of Science, Beijing 100049, China \\ ${ }^{4}$ Guangxi Key Laboratory of Hybrid Computation and IC Design Analysis, Guangxi University for Nationalities, \\ Nanning 530006, China \\ Correspondence should be addressed to Honggang Qi; hgqi@ucas.ac.cn and Anping He; hapetis@gmail.com
}

Received 11 February 2014; Accepted 20 March 2014; Published 14 April 2014

Academic Editor: Xiaoyu Song

Copyright (C) 2014 Daxin Tian et al. This is an open access article distributed under the Creative Commons Attribution License, which permits unrestricted use, distribution, and reproduction in any medium, provided the original work is properly cited.

We propose a bio-inspired model for making handover decision in heterogeneous wireless networks. It is based on an extended attractor selection model, which is biologically inspired by the self-adaptability and robustness of cellular response to the changes in dynamic environments. The goal of the proposed model is to guarantee multiple terminals' satisfaction by meeting the QoS requirements of those terminals' applications, and this model also attempts to ensure the fairness of network resources allocation, in the meanwhile, to enable the QoS-oriented handover decision adaptive to dynamic wireless environments. Some numerical simulations are preformed to validate our proposed bio-inspired model in terms of adaptive attractor selection in different noisy environments. And the results of some other simulations prove that the proposed handover scheme can adapt terminals' network selection to the varying wireless environment and benefits the QoS of multiple terminal applications simultaneously and automatically. Furthermore, the comparative analysis also shows that the bio-inspired model outperforms the utility function based handover decision scheme in terms of ensuring a better QoS satisfaction and a better fairness of network resources allocation in dynamic heterogeneous wireless networks.

\section{Introduction}

Recently, with the highly developed wireless communication technologies such as the wireless LANs (WLAN IEEE $802.11 \mathrm{a} / \mathrm{b} / \mathrm{g} / \mathrm{n} / \mathrm{p}$ standards), WiMAX (IEEE $802.16 \mathrm{a} / \mathrm{e}$ standards), the third and fourth generation cellular wireless (3G or $4 \mathrm{G}$ ), and satellite communications, substantial significant infrastructure of these wireless networks has been deployed to support the dramatically increasing demand for mobile terminals' access to network services anywhere and anytime. However, no single wireless access technology can be efficient to guarantee various users' demands for reliable connection and quality of service (QoS) over all situations. Consequently, the next generation wireless communication system is evolving, which depends on those heterogeneous wireless networks. In the nowadays heterogeneous wireless networks, multiple wireless access technologies as well as multiple radios have to interwork and to be used in a cooperative manner to realize the "always best connected" (ABC) concept in terms of high level of QoS satisfaction and fairness resources allocation [1].

Handover decision is one of the most important issues (including handover management, resource allocation, and mobility support) related to the heterogeneous wireless networks and should be efficiently addressed for the realization of the envisioned next generation communication system [2]. However, there exist some significant challenges in developing the essential functional components and in designing the corresponding algorithms for handover decision such as impracticality of centralized control, dynamic nature, resources constraint, and heterogeneity in the heterogeneous wireless environment [3]. 
On the other hand, the bio-inspired paradigm provides a novel approach to design a new powerful solution for many engineering problems $[4,5]$. Similar challenges met by the heterogeneous wireless communication system arising from dynamic nature, system complexity, heterogeneous architectures, and absence of centralized control have been well addressed by the biological system [6]. Many biological mechanisms such as the adaptability to environmental changes, inherent robustness to external perturbation, and self-optimization are appealing to be introduced in the handover decision solution to deal with those aforementioned significant challenges. As some interdisciplinary studies have argued, many biological mechanisms resulting from the evolution of nature over millions of years always go far away beyond the traditional technologies so that they are promising to be used to settle some complex engineering problems [7-9].

The attractor selection is one type of bio-inspired mechanism that induces cellular gene expression to adaptively respond to the dynamically changing environment. Its related model, that is, attractor selection model [10], has attracted much attention and has been extended to be implemented in many engineering domains consequently. For example, it has been applied in the robust robot control [11], the errortolerant wireless sensor networks control [12], the adaptive virtual network topology control [13], the adaptive routing protocol in mobile ad hoc networks or overlay networks [14], and the resources allocation among multiple users and multiple applications in the heterogeneous wireless environment [15].

Motivated by the attractor selection of cellular gene network, we adopt this bio-inspired mechanism for modeling the vertical handover decision in heterogeneous wireless networks. The goal of the paper is to deal with the varying heterogeneous wireless environmental conditions, at the same time, to guarantee users' satisfaction level, and to ensure the fairness of network resources allocation among multiple mobile terminals. We extend the basic attractor selection model which has been proposed in [10] to a novel one with a higher dimension for multiple attributes decision making. The upper and lower bounds of QoS requirements of multiple applications are combined with the dynamic wireless network conditions including bandwidth, end-to-end delay, and packet loss ratio to formulate a function for evaluating the terminal's QoS satisfaction. This utility function is used to assist the bioinspired model in performing the attractor selection mechanism. And the handover decision induced by the attractor selection mechanism allows us to capture the dynamic nature of the heterogeneous wireless environment and to evaluate the goodness of accessing wireless networks, so as to enable the handover decision adaptive to the wireless environmental changes.

The remainder of this paper is organized as follows. Section 2 formulates the handover decision problem to be solved and introduces the basic attractor selection model so as to extend it for making handover decision. The QoSoriented handover decision framework and the detailed scheme based on the proposed bio-inspired handover model are described in Section 3. Section 4 demonstrates some comparative simulation results and gives the analysis of our handover decision scheme. Finally, Section 5 concludes this work.

\section{System Model}

In this section, the core problem to be solved in the handover decision is described firstly, and then the basic attractor selection model as well as its corresponding biomechanism is presented. Following the mathematical form of the basic attractor selection model, we extend it to a novel form and apply this extended model for multiple attributes decision making.

2.1. Problem Formulation. We assume that each mobile terminal moving in a given heterogeneous wireless environment is equipped with a multimode communication device. These terminals with multiple wireless interfaces are able to access different wireless networks simultaneously. Namely, in this assumption, a mobile user is allowed to assign different wireless links to its different applications that are running in the terminal device. We consider a heterogeneous wireless environment composed of a network set of multiple heterogeneous wireless networks. We denote this network set as NetSet $=\left\{\right.$ net $_{1}$, net $_{2}, \ldots$, net $\left._{M}\right\}$. The parameter $M$, here, denotes the total number of those considered wireless networks. And then we consider that there are totally $Q$ vehicular terminals moving in this given heterogeneous wireless environment. All of these mobile terminals compose a set that is denoted by User $=\left\{u_{1}, u_{2}, \ldots, u_{Q}\right\}$, and each one $u_{k} \in$ User has a certain number of applications running in its terminal device. For instance, we denote those applications simultaneously running in $u_{k}$ as a set $S_{u_{k}}=\left\{s_{1}, s_{2}, \ldots, s_{N}\right\}$. Thus, each $u_{k}$ is required to make a decision to select the most suitable wireless network for each of its applications. Each application $s_{i} \in S_{u_{k}}$ may connect to the same network or may use different wireless links. Each $u_{k}$ performs the handover decision process during every discrete period $\Delta t$. When the network selection is done, the wireless interface of each application in $u_{k}$ is switched from the previous one to the new determined network.

2.2. Basic Attractor Selection Model. The basic attractor selection model is inspired from cell biology. It is used to describe the adaptive response of the gene expression in an Escherichia coli (E. coli) cell to the changes in its available nutrients, especially when there is no enough molecular machinery for signal transduction from the environment to the DNA expression [10]. The basic mathematical model of the attractor selection can be expressed by two nonlinear differential equations with stochastic noise as follows:

$$
\begin{aligned}
& \frac{d m_{1}}{d t}=\frac{S(A)}{1+\left(m_{2}\right)^{2}}-D(A) \times m_{1}+\eta_{1}, \\
& \frac{d m_{2}}{d t}=\frac{S(A)}{1+\left(m_{1}\right)^{2}}-D(A) \times m_{2}+\eta_{2},
\end{aligned}
$$

where $m_{1}$ and $m_{2}$ represent two different mRNA concentrations, respectively, corresponding to two different nutrients. 
$S(A)$ and $D(A)$ are, respectively, two different rate coefficients of nutrient synthesis and degradation. They are defined as the monotonously increasing function of cellular activity that is represented by the parameter $A$. In [10], $S(A)=6 A /(A+2)$ and $D(A)=A \cdot \eta_{1}$ and $\eta_{2}$ represent independent Gaussian white noise which is inherent in cellular gene expression.

From the viewpoint of dynamics system, the variable pair $\left[m_{1}, m_{2}\right]^{T}$ can be treated as a state of cellular metabolic phenotype and (1) essentially represents a cell dynamics system. An attractor is a stable state of the dynamics system to which the phase space trajectory of the system will converge, no matter what the initial conditions are. In fact, fluctuation inherently exists in the actual biological system, so the gene expression or other behaviors of a biological entity are not purely deterministic. Even though the state of the cellular dynamics system is perturbed by stochastic noise arising from the external environmental fluctuations, the system is able to gradually come to stability over time and finally stays at a new growth rate as well as at a well living state. The adaption of a cell to changes in its survival environment is analogous to the attractor selection of the dynamics system given in (1), in which the system will select and switch to a new stable and suitable state when the environmental conditions have been changed or perturbed to be unsuitable for previous state.

In addition, the cellular activity $A$ is an important parameter that lumps the fitness of the environmental conditions for the cell's survival to a single real value and it ranges from 0 to 1 . This parameter is used to comprehensively reflect the information of the cell's external environment, control the influence of the noise on the behavior of the dynamics system, and capture the phenotypic consequence that enables the cellular adaptation.

2.3. Extended Attractor Selection Model. Following the basic attractor selection model, we would like to introduce the appealing bio-inspired attractor selection mechanism to decision making under varying conditions in terms of improving the robustness and adaptability of the decision solution. In order to select the most appropriate wireless access network net $_{j} \in$ NetSet for any one application $s_{i} \in S_{u_{k}}$ that is running in the terminal device of a user $u_{k} \in$ User, we firstly define a decision vector as $\mathbf{X}^{s_{i}}(t)=\left[x_{\text {net }_{j}}^{s_{i}}(t)\right]_{\text {net }_{j} \in \text { NetSet }}=$ $\left[x_{\text {net }_{1}}^{s_{i}}(t), x_{\text {net }_{2}}^{s_{i}}(t), \ldots, x_{\text {net }_{M}}^{s_{i}}(t)\right]^{T}$, for each application $s_{i} \in S_{u_{k}}$. Each state value $x_{\text {net }_{j}}^{s_{i}}(t)$ in this decision vector refers to the score or the goodness of the network net ${ }_{j}$ relevant to the application $s_{i}$ at time $t$. Therefore, any $u_{k} \in$ User should maintain a set of $\left|S_{u_{k}}\right|$ decision vectors, since it has $\left|S_{u_{k}}\right|$ applications (it should be noted that the notation $\left|S_{u_{k}}\right|$ represents the number of elements in the set $S_{u_{k}}$ ). Then, we use these decision vectors to construct a matrix as follows:

$$
\begin{aligned}
& {\left[\mathbf{X}^{s_{1}}(t), \mathbf{X}^{s_{2}}(t), \ldots, \mathbf{X}^{s_{N}}(t)\right]} \\
& =\left[\begin{array}{cccc}
x_{\text {net }_{1}}^{s_{1}}(t) & x_{\text {net }_{1}}^{s_{2}}(t) & \cdots & x_{\text {net }_{1}}^{s_{N}}(t) \\
x_{\text {net }_{2}}^{s_{2}}(t) & x_{\text {net }_{2}}^{s_{2}}(t) & \cdots & x_{\text {net }_{2}}(t) \\
\vdots & \vdots & \ddots & \vdots \\
x_{\text {net }_{M}}^{s_{1}}(t) & x_{\text {net }_{M}}^{s_{2}}(t) & \cdots & x_{\text {net }_{M}}^{s_{N}}(t)
\end{array}\right] .
\end{aligned}
$$

The matrix shown in (2) is called a "decision matrix" or a "score matrix," whose rows index different wireless networks and columns index different applications. Each component value $x_{\text {net }_{j}}^{s_{i}}(t)$ in this decision matrix indicates the proportion of selecting the wireless network net ${ }_{j}$ as the target network for supporting the application $s_{i}$ at time $t$. Furthermore, once the decision matrix can be obtained, we can use (3) to determine the target network for the application $s_{i}$ :

$$
\text { net }^{*}\left(s_{i}\right)=\underset{\text { net }_{j} \in \text { NetSet }_{\text {et }}}{\operatorname{argmax}}\left\{x_{\text {net }_{j}}^{s_{i}}(t)\right\} .
$$

Aiming to update the state of the decision matrix, we here extend the basic attractor selection model to the new form with a higher dimension. We propose the novel extended model as follows:

$$
\begin{aligned}
\frac{d x_{\text {net }_{j}}^{s_{i}}(t)}{d t}= & \frac{\operatorname{syn}(\alpha)}{1+\left[x_{\text {net }^{*}\left(s_{i}\right)}^{s_{i}}(t)-x_{\text {net }_{j}}^{s_{i}}(t)\right]^{2}} \\
& -\operatorname{deg}(\alpha) \times x_{\text {net }_{j}}^{s_{i}}(t)+\eta_{\text {net }_{j}}^{s_{i}}(\mu, \sigma),
\end{aligned}
$$

where $s_{i} \in S_{u_{k}}$, net ${ }_{j} \in$ NetSet and $x_{\text {net }^{*}\left(s_{i}\right)}^{s_{i}}(t)$ is the maximum state value in the decision vector $\mathbf{X}^{s_{i}}(t)$ corresponding to the application $s_{i} \cdot \eta_{\text {net }_{j}}^{s_{i}}(\mu, \sigma)$ denotes the Gaussian white noise whose mean value is $\mu$ and whose standard deviation is $\sigma$. According to the basic attractor selection model, $\operatorname{syn}(\alpha)$ and $\operatorname{deg}(\alpha)$ should be designed as the monotonously increasing functions of the activity $\alpha$. Similar to [14], we adopt the polynomial form to formulate $\operatorname{syn}(\alpha)$ and directly set $\operatorname{deg}(\alpha)$ identical to $\alpha$ as follows:

$$
\begin{gathered}
\operatorname{syn}(\alpha)=\alpha \times\left(\beta \times \alpha^{n}+m\right), \\
\operatorname{deg}(\alpha)=\alpha,
\end{gathered}
$$

where $\beta$ and $m$ are both the positive real values and $n$ is a positive integer. On the basis of (4), we can use the model defined in (5) to dynamically and self-adaptively update the decision matrix defined in (2). Thus, based on the decision matrix, the handover decision can be made in terms of guaranteeing the terminal QoS satisfaction and selfadaptability.

2.4. Model Validation and Discussion. To analyze the proposed model given by (4), we split the stochastic nonlinear differential equation into two parts, that is, the deterministic term and the stochastic term. We define the notation $\Phi_{\text {net }_{j}}^{s_{i}}(\alpha, t)$ to represent the deterministic term

$$
\begin{aligned}
\Phi_{\text {net }_{j}}^{s_{i}}(\alpha, t)= & \frac{\operatorname{syn}(\alpha)}{1+\left[x_{\text {net }^{s_{i}}\left(s_{i}\right)}^{s_{i}}(t)-x_{\text {net }_{j}}^{s_{i}}(t)\right]^{2}} \\
& -\operatorname{deg}(\alpha) \times x_{\text {net }_{j}}^{s_{i}}(t) .
\end{aligned}
$$

$\Phi_{\text {net }_{j}}^{s_{i}}(\alpha, t)$ is a complex multivariate function of the deterministic parameters $\alpha$ and $t$. Following the notation above, we 
then reshape the extended attractor selection model defined in (4) into a more simple formulation which is composed of the deterministic term $\Phi_{\text {net }_{j}}^{s_{i}}(\alpha, t)$ and the stochastic term $\eta_{\text {net }_{j}}^{s_{i}}(\mu, \sigma)$ :

$$
\frac{d x_{\text {net }_{j}}^{s_{i}}(t)}{d t}=\Phi_{\text {net }_{j}}^{s_{i}}(\alpha, t)+\eta_{\text {net }_{j}}^{s_{i}}(\mu, \sigma) .
$$

From (7), it can be obviously observed that if the value of the activity $\alpha$ decreases due to some changes in the external conditions of this dynamics system, the magnitude of the deterministic term $\Phi_{\text {net }_{j}}^{s_{i}}(\alpha, t)$ will become smaller and it may decreasingly approach the magnitude of the stochastic term $\eta_{\text {net }_{j}}^{s_{i}}(\mu, \sigma)$, which means that $\left|\Phi_{\text {net }_{j}}^{s_{i}}(\alpha, t)\right| \approx$ $\left|\eta_{\text {net }_{j}}^{s_{i}}(\mu, \sigma)\right|$. In this situation, (7) tells us that the influence of the random noise becomes relatively enhanced and the behavior of the dynamics system is expected to be mostly dominated by the randomness of $\eta_{\text {net }_{j}}^{s_{i}}(\mu, \sigma)$. On the other hand, when the activity $\alpha$ increases to make the magnitude of the deterministic term $\Phi_{\text {net }_{j}}^{s_{i}}(\alpha, t)$ much larger than that of the stochastic term $\eta_{\text {net }_{j}}^{s_{i}}(\mu, \sigma)$, the deterministic term will govern this system so that its phase trajectory can asymptotically approach a more stable state against the fluctuation resulting from stochastic noise. At this point, the process of tending to a stable state can be analogous to the adaptive attractor selection in the gene expression of cells, since the dynamics of gene expression switches between different patterns, which is as well influenced by a combination of the deterministic and the stochastic behavior of cellular system.

Additionally, when the stochastic term $\eta_{\text {net }_{j}}^{s_{i}}(\mu, \sigma)$ is assumed to be zero, we can easily obtain the deterministic maximum value of the system state variable by setting $\Phi_{\text {net }_{j}}^{s_{i}}(\alpha, t)=0$ and net $_{j}=$ net $^{*}\left(s_{i}\right)$ as follows:

$$
\begin{aligned}
& \Phi_{\text {net }_{j}}^{s_{i}}(\alpha, t) \\
& =\frac{\operatorname{syn}(\alpha)}{1+\left[x_{\text {net* }^{s_{i}}\left(s_{i}\right)}(t)-x_{\text {net }_{j}}^{s_{i}}(t)\right]^{2}}-\operatorname{deg}(\alpha) \times x_{\text {net }_{j}}^{s_{i}}(t) \\
& \quad=\operatorname{syn}(\alpha)-\operatorname{deg}(\alpha) x_{\text {net }^{s_{i}}\left(s_{i}\right)}(t)=0 .
\end{aligned}
$$

Therefore, the deterministic state of maximum value at time $t$ derived from (8) is

$$
x_{\text {net }^{*}\left(s_{i}\right)}^{s_{i}}(t)=\frac{\operatorname{syn}(\alpha)}{\operatorname{deg}(\alpha)} .
$$

In the simple case, with considering the specific formulations of $\operatorname{syn}(\alpha)$ and $\operatorname{deg}(\alpha)$ given by (5), we can get the closedform expression for $x_{\text {net }^{*}\left(s_{i}\right)}(t)$ as

$$
x_{\max }^{s_{i}}=\frac{\alpha\left(\beta \times \alpha^{n}+m\right)}{\alpha}=\beta \times \alpha^{n}+m,
$$

where we use the notation $x_{\max }^{s_{i}}$ to represent $x_{\text {net }^{*}\left(s_{i}\right)}^{s_{i}}(t)$ for simplicity.
As mentioned before, we consider that these parameters $\beta, n$, and $m$ are positive real values. Thus, from (10), $x_{\max }^{s_{i}}$ is an increasing function of the activity $\alpha$. This means that when $\alpha$ is increased, the maximum system state is also increased along with this increasing $\alpha$. From the biological perspective, if cells successfully express their genes that are able to make them well survive and optimally grow in an uncertain and highly dynamic environment, their activity is consequently expected to approach the best level. It can be said that those cells select the adaptive attractor that corresponds to their suitable gene expression pattern. From this point, the level of the maximum system state given by (10) can capture the fitness degree of the dynamics system in the dynamic environment once the activity $\alpha$ is correlated with the varying environmental conditions. This also explains that it is appropriate to adopt the form of increasing function to represent $\operatorname{syn}(\alpha)$ and $\operatorname{deg}(\alpha)$.

Next, we come to consider the influence of random noise on the dynamics system. In fact, it is impossible to achieve the aforementioned selection of different attractors when only considering the deterministic term $\Phi_{\text {net }_{j}}^{s_{i}}(\alpha, t)$ in the dynamics system defined in (7). The biological system is always evolving along with inherent randomness. As $[16,17]$ have discussed, the stochastic fluctuation in cell systems is one of the most significant factors to drive the process of gene expression switching between attractor states. Some numerical simulations are done to validate how the deterministic and the stochastic terms affect the behavior of the dynamics system represented by the extended attractor selection model.

Following the basic attractor selection model defined in (1), we adopt the assumption of Gaussian white noise for the stochastic term $\eta_{\text {net }_{j}}^{s_{i}}(\mu, \sigma)$, and then without loss of generality we set $\mu=0$ in the following experiments. In the simulation, the standard deviation $\sigma$ is, respectively, set to be $0.5,1$, and 2.5, and the parameters $\beta, n$, and $m$ are fixed at 5, 3, and 5, respectively. These simulations allow us to perform comparative analysis and investigate the properties of the bio-inspired model in the dynamic environment of different stochastic perturbation magnitudes.

Furthermore, because our goal here is to investigate the properties of the proposed model in terms of different random noises, without loss of generality we consider the specific form of the model with only three state variables, respectively, denoted by $x_{1}, x_{2}$, and $x_{3}$ :

$$
\frac{d x_{i}}{d t}=\frac{\operatorname{syn}(\alpha)}{1+\left(x_{\max }-x_{i}\right)^{2}}-\operatorname{deg}(\alpha) \times x_{i}+\eta_{i}(0, \sigma),
$$

where $i=1,2,3$ and $x_{\max }=\max _{i=1,2,3}\left\{x_{i}\right\}$. Also, it is worth pointing out that the state variables are all restricted in the positive real number domain; that is, $x_{i} \geq 0$, for all $i=1,2$, and 3 ; since these state variables have actual meaning when they are related to the level of cells gene expression, we will reset them to 0 when any one state variable changes to be lower than zero.

Now, we set the simulation time from 0 seconds to 3600 seconds. And then we employ a changing environment in the simulations where a square wave with period 900 seconds is 


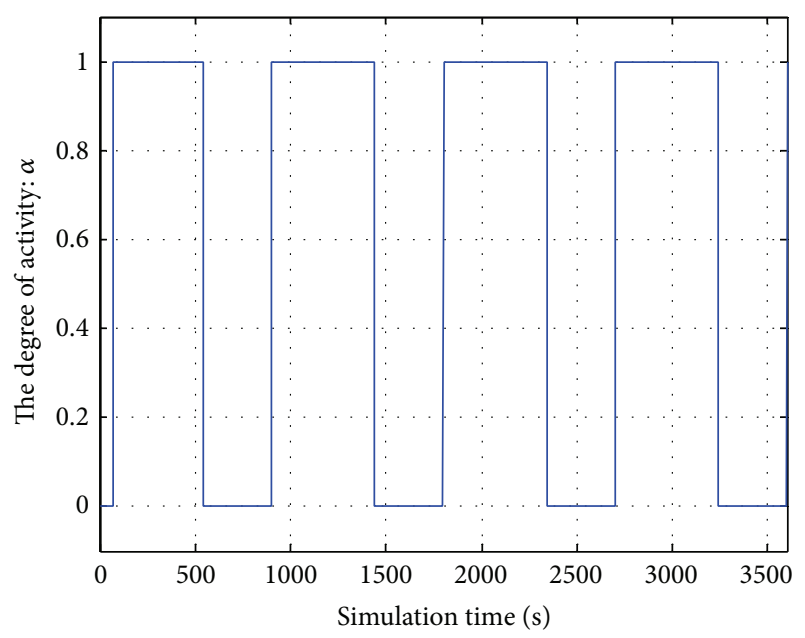

FIGURE 1: The varying activity $\alpha$ in simulations.

adopted to simulate the time series for the varying activity $\alpha$. The maximum and the minimum peaks of the square wave are set to be 1 and 0 , respectively, and its duty cycle (i.e., the percent of the period in which the signal is larger than 0 ) is set to be $60 \%$. The simulated varying activity $\alpha$ is given in Figure 1.

In Figure 2, the subgraphs (a), (b), and (c), respectively, show the variation of the dynamics system. In the first case, with the standard deviation of the random noise $\sigma=0.5$, the dynamics system composed of $x_{i}(i=1,2,3)$ stably stays at the attractor whose maximum state is $x_{2}$ and whose lower states are $x_{1}$ and $x_{3}$. It is because the magnitude of the stochastic term in the model is very small, while the deterministic term always dominates the behavior of the dynamics system even when the activity $\alpha$ decreases to 0 (see Figure 1). This means that the system is trapped into one attractor and will not switch to another. When the magnitude of the stochastic term increases (namely, the standard deviation of the random noise is set to be larger as shown in the cases of (b) and (c)), the state of the dynamics system fluctuates more fiercely with the low value of the activity $\alpha$. However, when the activity $\alpha$ jumps from the minimum to the maximum peak, the dynamics system always evolves to a stable state, that is, one attractor. For instance, in the subgraph (c), the system stays at the attractor whose maximum state value is $x_{1}$ and whose lower states are $x_{2}$ and $x_{3}$ during the simulation time interval which is from 70.5 seconds to 535 seconds (see Figure 1), whereas this system switches to another attractor whose maximum state value changes to be $x_{3}$ after a relatively shorter time interval (535, 908.3 seconds). From the experimental results, it is confirmed that the proposed bio-inspired model well inherits the mechanism of attractor selection and is able to dynamically capture the variation of the environmental conditions.

\section{QoS-Oriented Handover Decision}

This section gives the handover framework used in this work as well as the formulation for evaluating the terminal
QoS satisfaction. Following this, we also present the detailed QoS-based handover decision scheme based on the extended attractor selection model.

\subsection{Handover Decision Framework Based on the Extended} Attractor Selection Model. Based on the extended attractor selection model, we propose a distributed handover decision making scheme framework. This framework is outlined in Figure 3. At each time period $\Delta t$, each individual mobile terminal $u_{k} \in$ User can perform the handover decision process independently. Because the extended attractor selection model only needs the information on the QoS requirements of the applications that belong to an individual terminal and the information on the current wireless network conditions, multiple terminals do not need to exchange their decision information with each other. In our scheme, the handover decision is made at the terminal side instead of the network side. Therefore, it is not necessary to deploy a centralized control entity for managing the handover process.

Under the distributed handover decision framework shown by Figure 3, each application of a mobile terminal firstly provides its QoS requirements to its terminal during each time period. In the meanwhile, the mobile terminal needs to sense the current network conditions by making some signaling messages interact with the networks through air interface. Then the utility of each application is evaluated by using the proposed utility function (the utility function is developed in Section 3.2), that is, quantifying the degree in which the QoS requirements of each application are satisfied by its current wireless link. Based on the QoS satisfaction degree of each application, we evaluate the terminal QoS satisfaction so as to map the degree of the terminal's QoS satisfaction to the activity $\alpha$. Furthermore, the activity $\alpha$ is inputted to the extended attractor selection model and is used to drive the model to update the handover decision matrix given in (2) as well as determining the target networks by using (3). Finally, the handover can be done according to the determined target networks.

3.2. Quantification of Terminal's QoS Satisfaction. In this work, we take the upper and lower bounds of QoS requirements of each application into consideration. The QoSrelated attributes considered include bandwidth, end-to-end delay, and packet loss ratio. The bandwidth, delay, and packet loss ratio are, respectively, indexed by the notations $b, d$, and $p$. Based on this, we denote the upper bound of bandwidth, delay, and packet loss ratio required by the application $s_{i} \epsilon$ $S_{u_{k}}$ as $U\left(s_{i}, x\right)(x=b, d, p)$ and the lower bound of those requirements as $L\left(s_{i}, x\right)(x=b, d, p)$. We assume that the application $s_{i}$ is currently connected to the network net ${ }_{j} \epsilon$ NetSet. Additionally, the current conditions (including available bandwidth, transmission delay, and packet loss ratio) of the network net ${ }_{j}$ at time $t$ are denoted as $C\left(\right.$ net $\left._{j}, x, t\right)$ $(x=b, d, p)$. Then we evaluate the utility of each attribute 


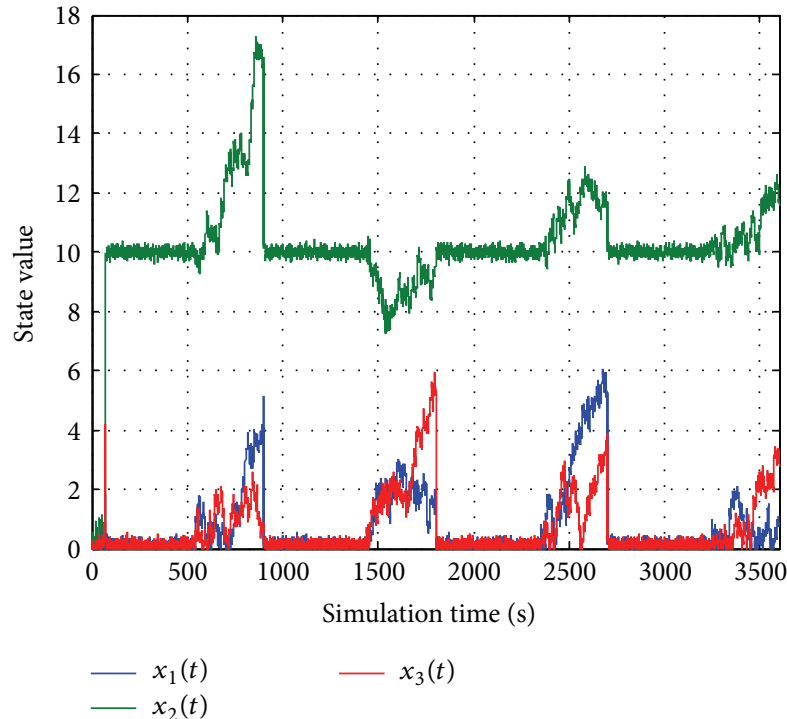

(a) $\sigma=0.5$

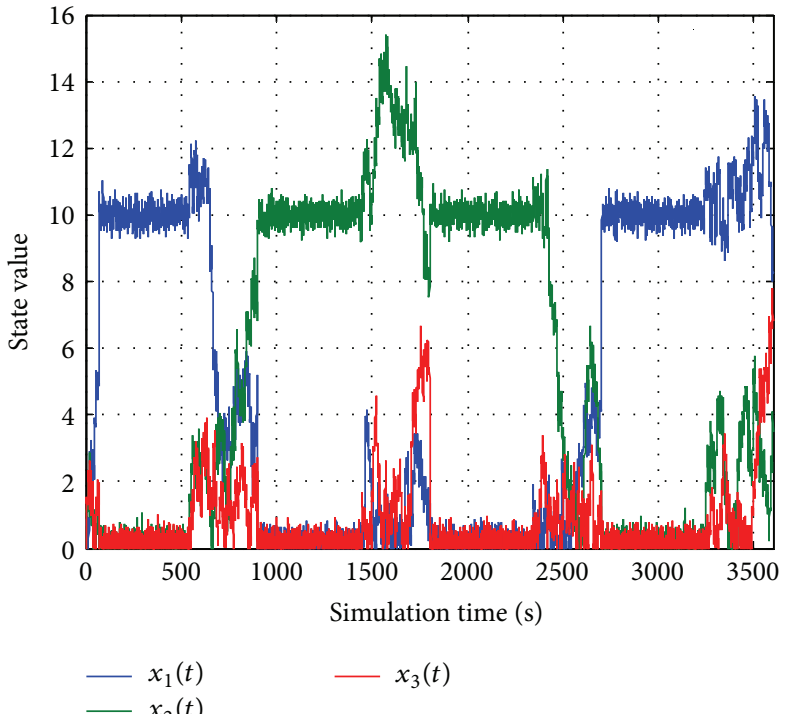

(b) $\sigma=1$

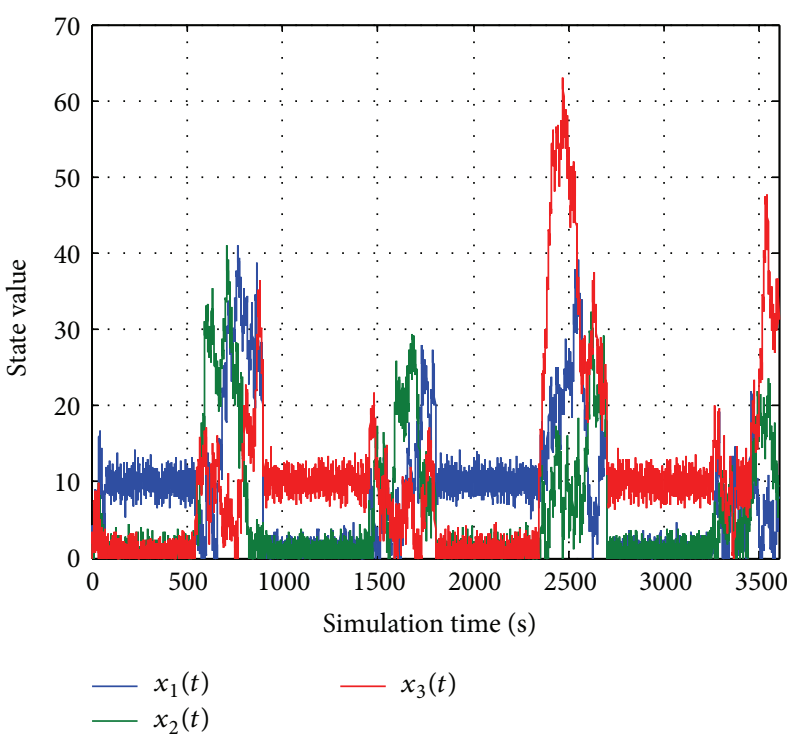

(c) $\sigma=2.5$

FIGURE 2: Evaluation of the impact of the random noise variability on the dynamics system.

perceived by the application $s_{i}$ with the linear normalization strategy as follows:

$$
\begin{array}{ll}
R\left(s_{i}, x, t\right) & \\
\quad=\frac{\min \left\{U\left(s_{i}, x\right), C\left(\text { net }_{j}, x, t\right)\right\}-L\left(s_{i}, x\right)}{U\left(s_{i}, x\right)-L\left(s_{i}, x\right)} ; & x=b, \\
R\left(s_{i}, x, t\right) & \\
\quad=\frac{U\left(s_{i}, x\right)-\max \left\{L\left(s_{i}, x\right), C\left(\text { net }_{j}, x, t\right)\right\}}{U\left(s_{i}, x\right)-L\left(s_{i}, x\right)} ; \quad x=d, p .
\end{array}
$$

In addition, we adopt the weighted sum method to lump different $R\left(s_{i}, x, t\right)(x=b, d, p)$ to one variable as follows:

$$
G\left(s_{i}, t\right)=\sum_{x=b, d, p} \omega\left(s_{i}, x\right) \times f\left(R\left(s_{i}, x, t\right)\right),
$$

where $\omega\left(s_{i}, x\right)$ is the positive weight corresponding to the QoS attribute $x(x=b, d, p)$, and they must satisfy the constraint $\sum_{x=b, d, p} \omega\left(s_{i}, x\right)=1 . f(\cdot)$ is a monotonously increasing function that can map $R\left(s_{i}, x, t\right)$ to $[0,1]$. In this work, we formulate the function as the sigmoid form:

$$
f(v)=\frac{1}{1+\exp \left(-c_{1} \times v+c_{2}\right)},
$$

where $c_{1}$ and $c_{2}$ are both positive real parameters. 


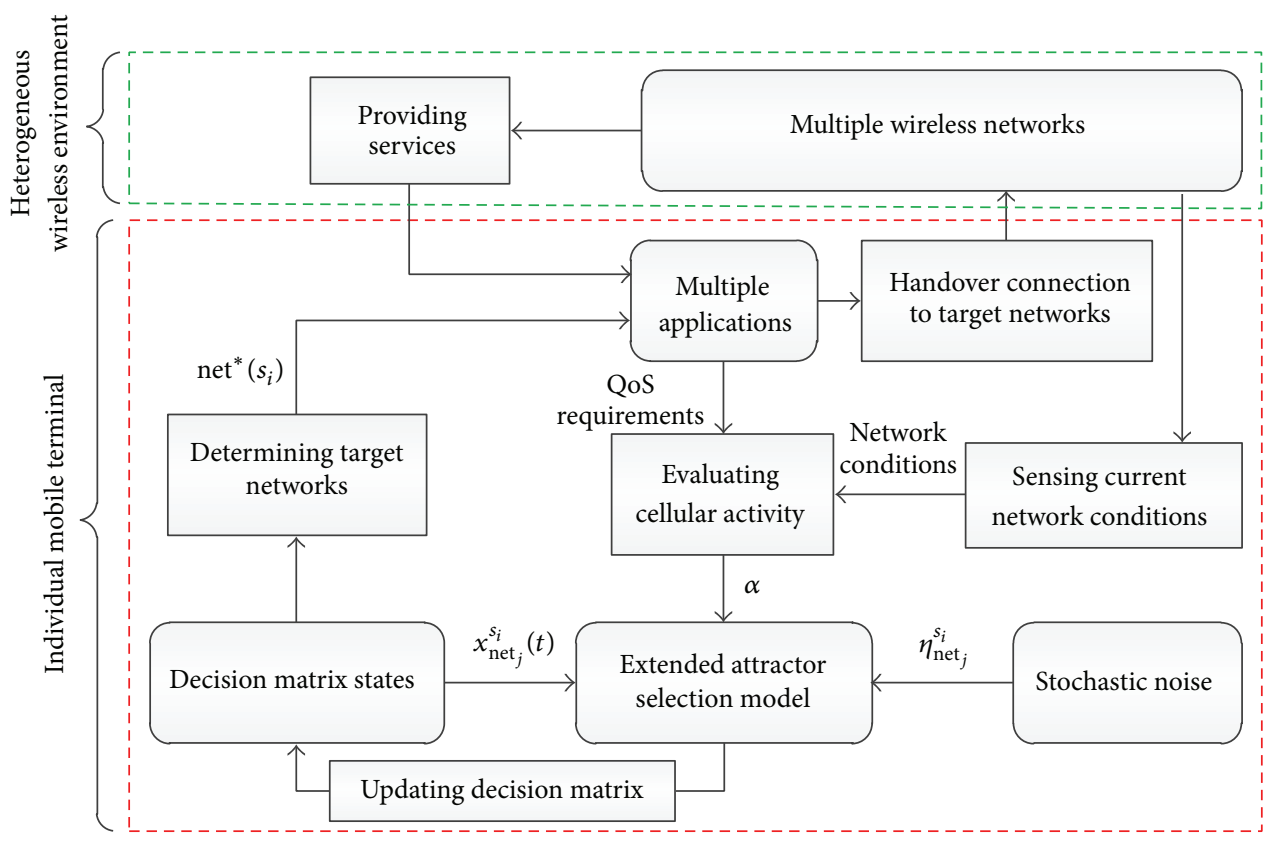

Figure 3: The handover decision framework based on the extended attractor selection model.

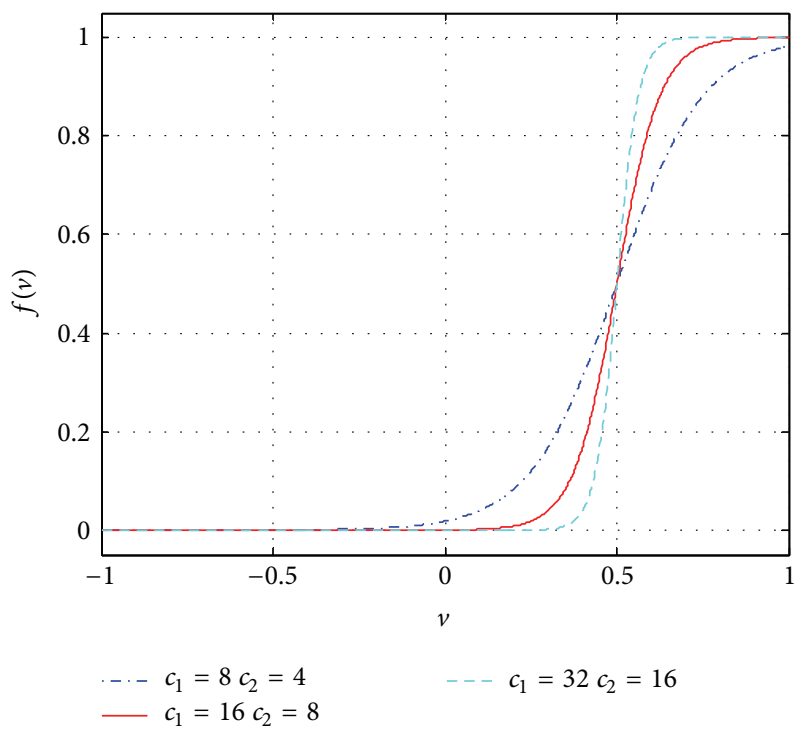

Figure 4: The variation of the adopted sigmoid function with different parameter settings.

In order to investigate the influence of $c_{1}$ and $c_{2}$ on $f(v)$ given in (14), we vary these parameters and plot the variation of the function with respect to different parameter settings. For simplicity but without loss of generality, we set $c_{1}=2 c_{2}$ and obtain the results shown in Figure 4. The figure shows the slope of $f(v)$ with considering different values of $c_{1}$ and $c_{2}$. As we can see, the larger the parameters $c_{1}$ and $c_{2}$ are, the steeper the slope of $f(v)$ becomes. And the function value can stably stay at a relatively low (or high) level when the variable $v$ approaches 0 (or 1 ). Because $R\left(s_{i}, x, t\right)$ represents the degree of the wireless network satisfying the application $s_{i}$ in terms of the requirements on bandwidth, transmission delay and packet loss ratio, its value may change abruptly due to the dynamic nature of the wireless environment. On the basis of the results of Figure 4 , we adopt $f(v)$ to map $R\left(s_{i}, x, t\right)$ to the interval $[0,1]$ and fix the parameters $c_{1}$ and $c_{2}$ at 16 and 8 , respectively, so that the weighted sum term $G\left(s_{i}, t\right)$ given in (13) is limited in $[0,1]$ and its sensitivity is inhibited when $R\left(s_{i}, x, t\right)$ becomes either too much small or large.

On the other hand, the function value $G\left(s_{i}, t\right)$ comprehensively represents the degree of the QoS satisfaction of the application $s_{i}$. In order to evaluate the QoS satisfaction of the terminal $u_{k}$, we combine all of the $G\left(s_{i}, t\right)\left(s_{i} \in S_{u_{k}}\right)$ with the cumulative product strategy and yield

$$
F\left(u_{k}, t\right)=\prod_{s_{i} \in S_{u_{k}}} G\left(s_{i}, t\right) .
$$

Since the terminal QoS satisfaction $F\left(u_{k}, t\right)$ may change suddenly at the instant time $t$ along with the varying wireless environment, it is not feasible to directly set the activity $\alpha$ equal to $F\left(u_{k}, t\right)$ as the input of the extended attractor selection model. We further adopt the weighted moving averaging method to map $F\left(u_{k}, t\right)$ to $\alpha$ as follows:

$$
\alpha=\frac{\int_{t-T}^{t} \lambda(\tau) \times F\left(u_{k}, \tau\right) d \tau}{T}
$$

where $T$ is the fixed time window $(0<T<t) . \lambda(\tau)(\tau \in$ $[t-T, t])$ is the time-dependent positive weight that is used to reflect the significance of $F\left(u_{k}, \tau\right)$ and needs to satisfy the constraint of $\int_{t-T}^{T} \lambda(\tau) d \tau / T=1 . \lambda(\tau)$ should be the increasing function of the time variable $\tau$, because the closer to the current instant $t$ the time variable $\tau$ is, the more significant 
the information reflected from $F\left(u_{k}, \tau\right)$ becomes. Therefore, for simplicity, we design $\lambda(\tau)$ as follows:

$$
\lambda(\tau)=\frac{2 \tau}{(2 t-T)} .
$$

It is easy to validate that (17) satisfies the constraint $\int_{t-T}^{T} \lambda(\tau) d \tau / T=1$ and $\lambda(\tau) \geq 0$.

Based on (16), we can suppress the sensitivity of the activity $\alpha$ to the fierce changes in the dynamic wireless environment. Once $\alpha$ is obtained by (16), we treat $\alpha$ as the external input of the extended attractor selection model. Consequently, the model is driven to update the decision matrix in (2) so as to make the handover decision adaptively and automatically. Since each individual mobile terminal can independently process the extended attractor selection model and can perform the handover decision according to the aforementioned distributed framework, each individual terminal is essentially analogous to a cellular system.

\section{Performance Evaluation}

We perform some comparative simulations to evaluate the proposed bio-inspired handover decision scheme in this section through a discrete event simulator that we have developed in MATLAB with the object-oriented programming. Firstly, we present a typical heterogeneous wireless environment as the simulation scenario. And then we present the performance evaluation of our bio-inspired scheme and compare our scheme with the typical utility function based scheme with the simple additive weighting (SAW) [18].

4.1. Simulation Scenario. We consider a vehicular heterogeneous wireless environment where there exist three types of wireless networks including $3 \mathrm{G}$ cellular network (WCDMA), WiFi (IEEE 802.11n), and DSRC (IEEE 802.11p). This simulation scenario is given in Figure 5. The cellular network and DSRC are both assumed to be able to coverage the expressway denoted by the segment $\mathrm{AE}$ whose length is equal to 1000 meters. There are totally $3 \mathrm{WiFi}$ access points (APs) deployed at the location of Points B, C, and D which are along the expressway AE. The coverage of WiFi APs is set to be 200 meters as illustrated in Figure 5. In the simulation, multiple vehicular terminals are stochastically generated and uniformly distributed on the expressway at the beginning of the simulation. The direction of these vehicles is from $\mathrm{A}$ to $\mathrm{E}$ and these vehicles are moving at a constant velocity during the simulation. In addition, each of these vehicular terminals runs three types of networking applications including the voice application, the video application, and the data stream. In addition, we set the time interval $\Delta t=0.5$ seconds as the discrete period during which the decision matrix is updated at a time through processing the extended attractor selection model.

4.2. Simulation Settings. In this simulation, we use CDMA based cellular network and IEEE 802.11 based WLAN access
TABLE 1: Network conditions settings.

\begin{tabular}{lccc}
\hline Network & $\begin{array}{c}\text { Capacity } \\
(\text { Mbyte/s) }\end{array}$ & $\begin{array}{c}\text { Delay } \\
(\mathrm{ms})\end{array}$ & $\begin{array}{c}\text { Packet loss ratio } \\
(\%)\end{array}$ \\
\hline Cellular & 1.3 & 25 & 0.08 \\
WiFi (AP1) & 25 & 8 & 0.04 \\
WiFi (AP2) & 25 & 25 & 0.04 \\
WiFi (AP3) & 25 & 45 & 0.04 \\
DSRC & 27 & 50 & 0.03 \\
\hline
\end{tabular}

networks including WiFi and DSRC. The network conditions related settings are referred to in the work [19] and are given in Table 1 .

It is worth pointing out that the network conditions are varying during simulation. Since the number of the connections to a network changes all the time and has a significant influence on the network resource, we assume that the amount of the connections to one network is the main factor to change this network's conditions over time. In order to simulate the dynamic nature of the heterogeneous wireless networks, we vary those network QoS attributes during simulation. Denote the amount of the applications that are currently connected to a network net ${ }_{j} \in$ NetSet at time $t$ as num $\left(\right.$ net $\left._{j}, t\right)$. For simplicity but without loss of generality, we simulate the time-dependent QoS attributes by using the following formulations:

$$
\begin{aligned}
& C\left(\text { net }_{j}, b, t\right)=\left\lfloor\frac{\operatorname{Capacity}\left(\text { net }_{j}\right)}{\operatorname{num}\left(\operatorname{net}_{j}, t\right)}\right\rfloor, \\
& C\left(\text { net }_{j}, d, t\right) \\
& =\operatorname{Delay}\left(\text { net }_{j}\right) \\
& \times\left[1+0.5 \times f\left(\frac{\operatorname{num}\left(\text { net }_{j}, t\right)}{\sum_{\text {net }_{j} \in \mathrm{NetSet}} \operatorname{num}\left(\text { net }_{j}, t\right)}\right)\right], \\
& C\left(\text { net }_{j}, p, t\right) \\
& =\text { Packet_loss_ratio }\left(\text { net }_{j}\right) \\
& \times\left[1+0.5 \times f\left(\frac{\operatorname{num}\left(\text { net }_{j}, t\right)}{\left.\sum_{\text {net }_{j} \in \text { NetSet }_{1}} \operatorname{num}_{\left(\text {net }_{j}, t\right)}\right)}\right),\right.
\end{aligned}
$$

where the notations Capacity $\left(\right.$ net $\left._{j}\right)$, Delay $\left(\right.$ net $\left._{j}\right)$, and Packet_loss_ratio(net $\left.{ }_{j}\right)$, respectively, present the capacity, delay, and packet loss ratio of the network net ${ }_{j}$ whose values are given in Table $1, f(\cdot)$ is also the sigmoid function as shown by (14), and $\lfloor\cdot\rfloor$ is the floor function. From (18), it is obvious that the more the amount of the users connected to a network is, the worse the performance of this network will become. Thus, we are allowed to simulate the dynamic nature of the wireless network in the experiments.

Furthermore, Table 2 gives three typical applications and the upper and lower bounds of their QoS requirements. Their parameters settings are referred to in [20]. In our 


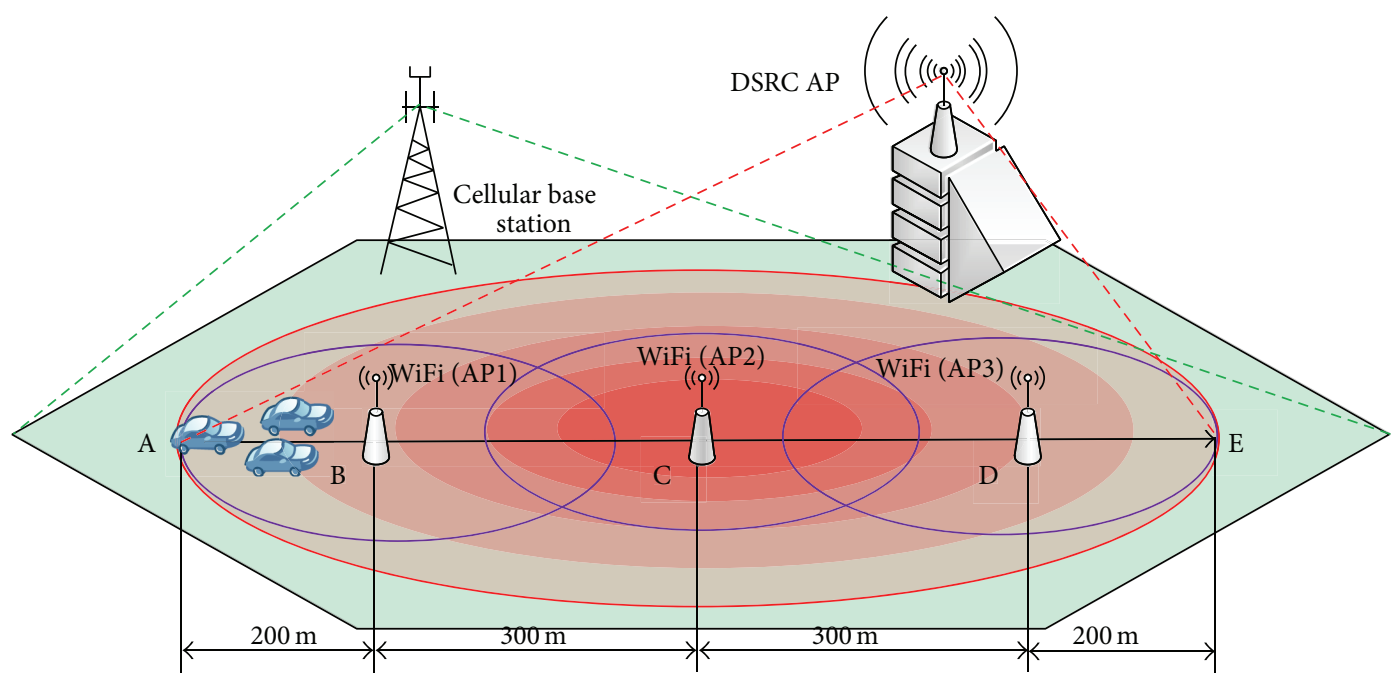

FIgURe 5: A simulation scenario.

TABLE 2: Applications settings.

\begin{tabular}{lcccc}
\hline QoS attributes & Requirements & Voice & Video & Data stream \\
\hline \multirow{2}{*}{ Bandwidth (Kbyte/s) } & $U\left(s_{i}, b\right)$ & 64 & 128 & 500 \\
& $L\left(s_{i}, b\right)$ & 9 & 30 & 128 \\
\hline \multirow{2}{*}{ Delay (ms) } & $U\left(s_{i}, d\right)$ & 150 & 150 & 120 \\
& $L\left(s_{i}, d\right)$ & 0 & 0 & 0 \\
\hline \multirow{2}{*}{ Packet loss ratio (\%) } & $U\left(s_{i}, p\right)$ & 0.08 & 0.03 & 0.08 \\
& $L\left(s_{i}, p\right)$ & 0 & 0 & 0
\end{tabular}

simulations, the total number of the applications run by each terminal is limited to three, and the applications of each terminal are generated from Table 2 at random. For example, a vehicular terminal may run all the three types of the applications including voice, video, and data stream while one other terminal may run two voice applications and a video application.

Additionally, we adopt the model settings illustrated in Table 3 for our extended attractor selection model. Since different applications are sensitive to different QoS attributes, the weights of the QoS attributes required by different applications are also different from each other. For instance, the voice application may require lower end-to-end delay and the video application needs more bandwidth for transmission. We use the detailed settings on $\omega\left(s_{i}, x\right)(x=b, d, p)$ given in Table 4 for our experiments.

4.3. Numerical Evaluation. In order to analyze the process of the handover decision driven by the attractor selection mechanism from the extended attractor selection model, we initially set the number of the total vehicular terminals to be equal to 90 and the initial velocity of those terminals is set to be $45 \mathrm{~km} / \mathrm{h}$. We randomly choose one of those terminals and illustrate its relevant simulation results in Figure 6. This vehicular terminal has two types of applications, one of which is the voice-related application and the other two are the video-related applications. Subgraphs (a), (b), and (c)
TABLE 3: Model settings.

\begin{tabular}{lc}
\hline Parameter & Value \\
\hline$\mu$ & 0 \\
$\sigma$ & 1 \\
$\beta$ & 5 \\
$m$ & 5 \\
$n$ & 3 \\
$c_{1}$ & 16 \\
$c_{2}$ & 8 \\
$T$ & $25 \mathrm{~s}$ \\
\hline
\end{tabular}

TABLE 4: Settings on the weights.

\begin{tabular}{ccccc}
\hline & $\omega\left(s_{i}, x\right)$ & \multicolumn{3}{c}{$x$} \\
& & $b$ (bandwidth) & $d$ (delay) & $p$ (packet loss ratio) \\
\hline \multirow{3}{*}{$s_{i}$} & Voice & 0.3 & 0.5 & 0.2 \\
& Video & 0.5 & 0.3 & 0.2 \\
\multicolumn{2}{c}{ Data stream } & 0.4 & 0.3 & 0.3 \\
\hline
\end{tabular}

in Figure 6, respectively, show the variation of the decision vector state corresponding to each application against the simulation time. For example, in subgraph (a), the blue dashed line plots the variation of $x_{\text {Celluar }}^{\text {voice }}(t)$ that represents the variation of the fitness of the cellular network for the voice application against the simulation time.

From these results, it is observed that the applications of this terminal are able to adapt its wireless access link according to the varying network conditions. According to subgraph (a), the voice application stably accesses to the cellular network during the whole simulation time, while the other two video applications switch their connections between different wireless networks in order to guarantee their QoS requirements. Even though these two applications are of the same type, their decision behaviors are different from each other. As shown in subgraphs (b) and (c), the accessing link of the 


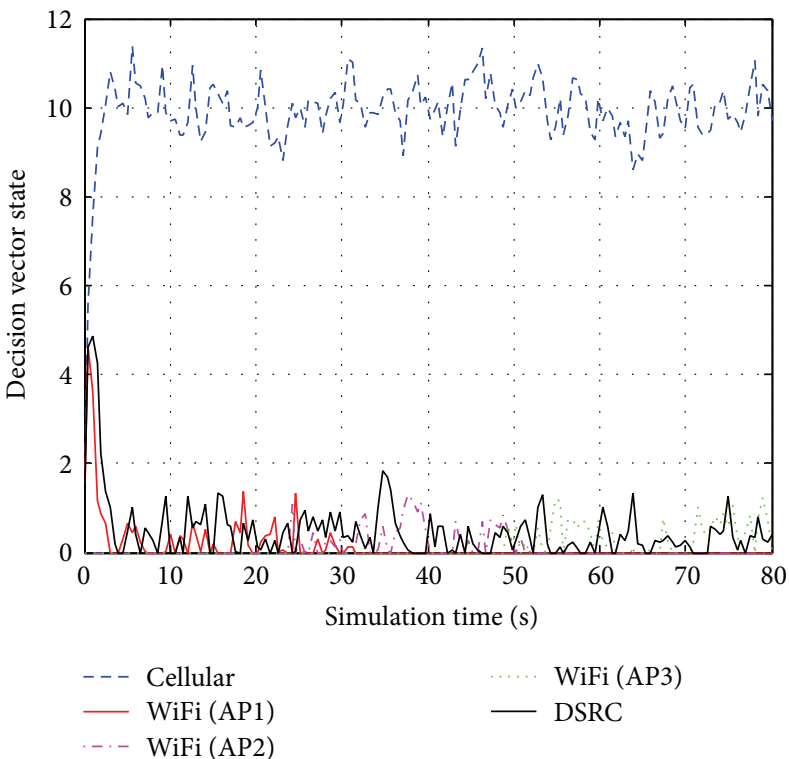

(a) The voice application

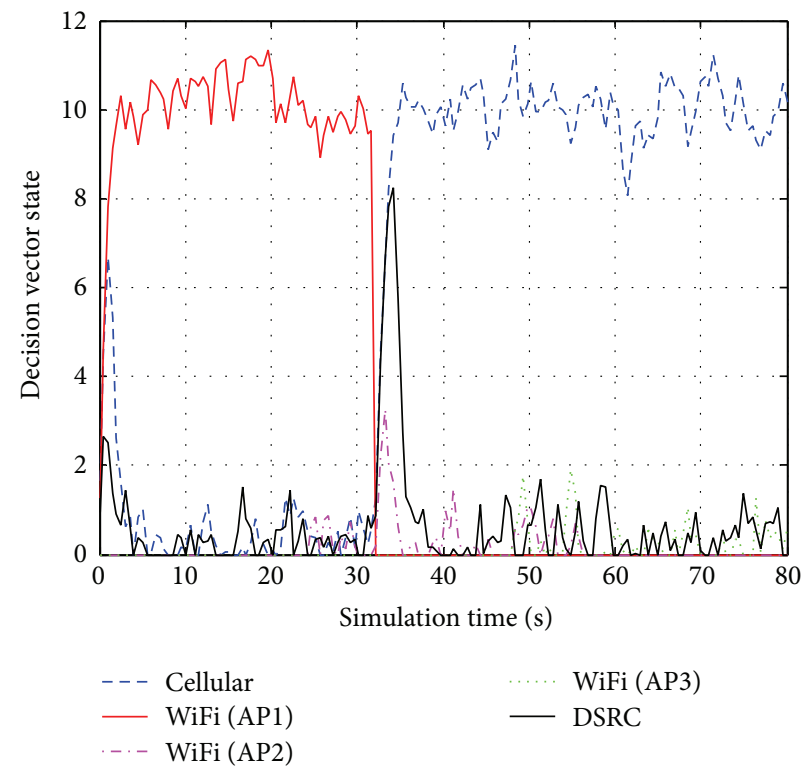

(b) One video application

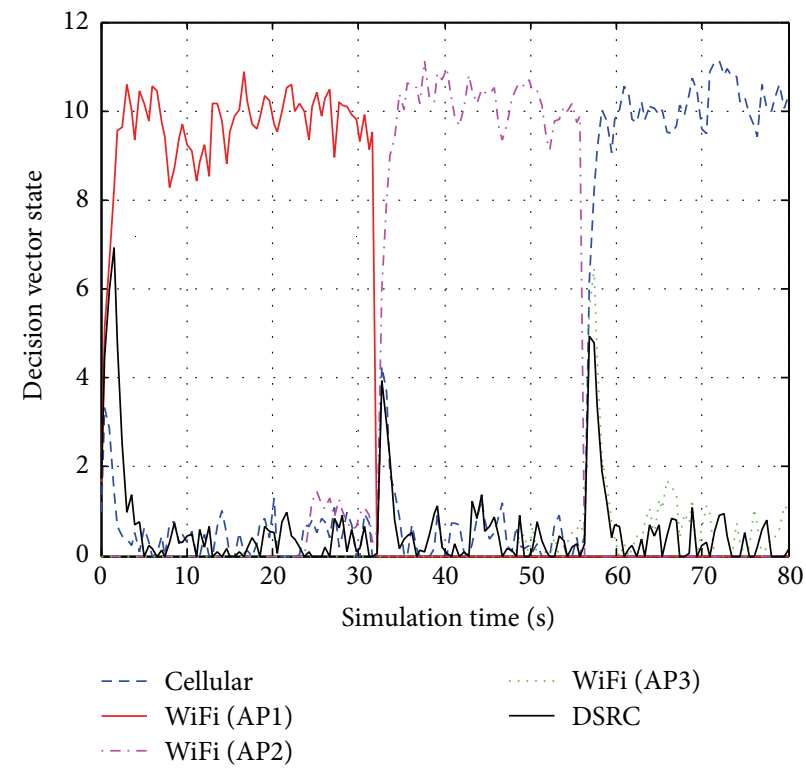

(c) Another video application

Figure 6: The variation of the decision vector states of the different applications.

first video application switches to the cellular network from the first WiFi network when this terminal moves out of the coverage of the first access point (marked as "WiFi (AP1)" in Figure 5), while the second video application switches its connection to the second WiFi network and maintains this wireless connection for a while; after that, it also selects and stably connects to the cellular network. Since the heterogeneous network conditions are changing all the time, this terminal selects different appropriate networks for each of its applications according to the real-time network conditions so that it can ensure well QoS satisfaction. The average activity of this terminal reaches 0.98962 . According to the definition of the activity illustrated by (16), the larger value of the activity indicates the higher degree of the terminal QoS satisfaction. Thus, this result implies that this terminal achieves a good QoS satisfaction during the process of handover decision making. 


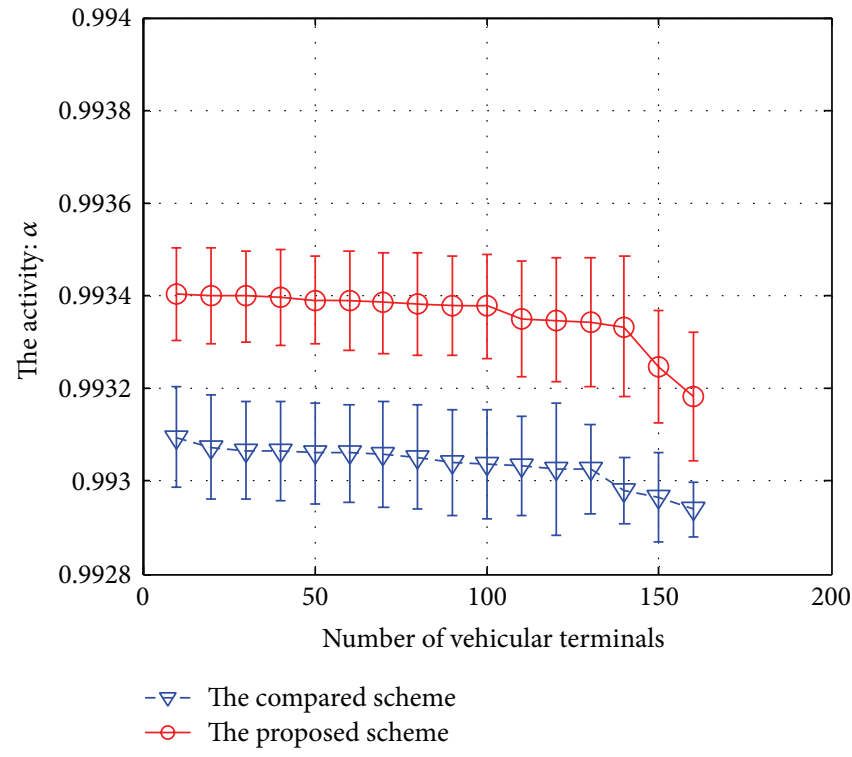

(a) The results of the activity $\alpha$

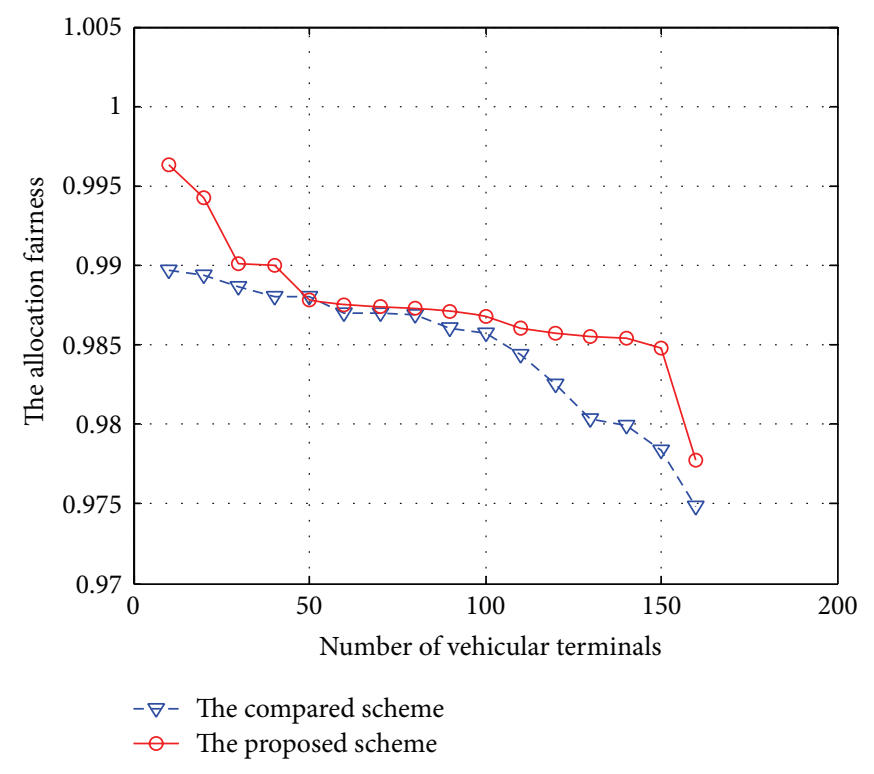

(b) The results of the fairness

Figure 7: The simulation results under different vehicular terminals.

Next, we compare the simulation results obtained by our proposed bio-inspired handover decision scheme with those obtained by the utility function based scheme with SAW. Additionally, in order to perform the comparative evaluation in terms of the fairness of network resources allocation, we refer to the concept of the fairness index of the resource allocation among multiple entities in [21] and then define the following equation for calculating the fairness metric:

$$
\text { The Allocation Fairness }=\frac{\left[\sum_{u_{k} \in \mathrm{User}} \bar{\alpha}\left(u_{k}\right)\right]^{2}}{\mathrm{Q} \times\left[\sum_{u_{k} \in \mathrm{User}}\left(\bar{\alpha}\left(u_{k}\right)\right)^{2}\right]},
$$

where $Q$ is the number of the total vehicular terminals and $\bar{\alpha}\left(u_{k}\right)$ is the average activity corresponding to $u_{k}$ that can be calculated by averaging all the values of the activity obtained at every time period $\Delta t$.

Firstly, we comparatively analyze the results of the two schemes under the specific simulation condition where the initial velocity is set to be $45 \mathrm{~km} / \mathrm{h}$ and the number of total vehicular terminals discretely ranges from 10 to 160 so as to simulate the specific scenarios of different traffic densities. We calculate the average value and standard deviation of the activity $\alpha$ per individual terminal against different total numbers of vehicular terminals and the fairness of the overall network resources allocation according to (19). These results are illustrated in Figure 7. Since the amount of total vehicular terminals increases and the overall network resources are limited, the competition among multiple terminals becomes much fiercer so that the terminal QoS satisfaction and the fairness metric obtained by both schemes decreases along with increasing the amount of terminals. However, from subgraphs (a) and (b) in Figure 7, it can be found that the proposed scheme achieves a better activity and a better fairness of network resources allocation on average when compared with the results obtained by the compared scheme.

Furthermore, we perform the comparative simulations under different initial terminal velocity. We fix the number of vehicular terminals at 100 and vary the terminal velocity. The initial velocity is discretely set to be $15 \mathrm{~km} / \mathrm{h}, 45 \mathrm{~km} / \mathrm{h}$, $75 \mathrm{~km} / \mathrm{h}$, and $100 \mathrm{~km} / \mathrm{h}$ so as to simulate different mobility scenarios. We also calculate the average value and standard deviation of the activity $\alpha$ per individual terminal against different initial terminal velocity as well as the average value of the fairness metric. Figure 8 demonstrates those numerical results. Because the faster the vehicular terminal moves the shorter the duration when the applications of each terminal maintain their wireless links will last; a relatively high mobility may increase the times of switching wireless connection. This will reduce the efficiency of network resources allocation. Thus, the performance of both handover decision schemes degrades along with increasing the velocity. On the other hand, as shown in Figure 8, the activity on average obtained by our proposed scheme is larger than the compared scheme. Furthermore, the average degree of the fairness of the network resources allocation obtained by our scheme is larger than the compared scheme.

In fact, the utility function based handover decision with SAW selects the access link for each application in a deterministic manner. It ranks the networks based on the terminal utility and attempts to maximize every terminal's QoS satisfaction. Under the distributed framework, each application of these terminals tends to access the best network. Then, when large amount of applications accesses the same best network at the same time, this network will become congested and its performance will deteriorate rapidly. Consequently, this network becomes nonoptimal and its users will again switch to another same best network simultaneously. Thus, multiple 


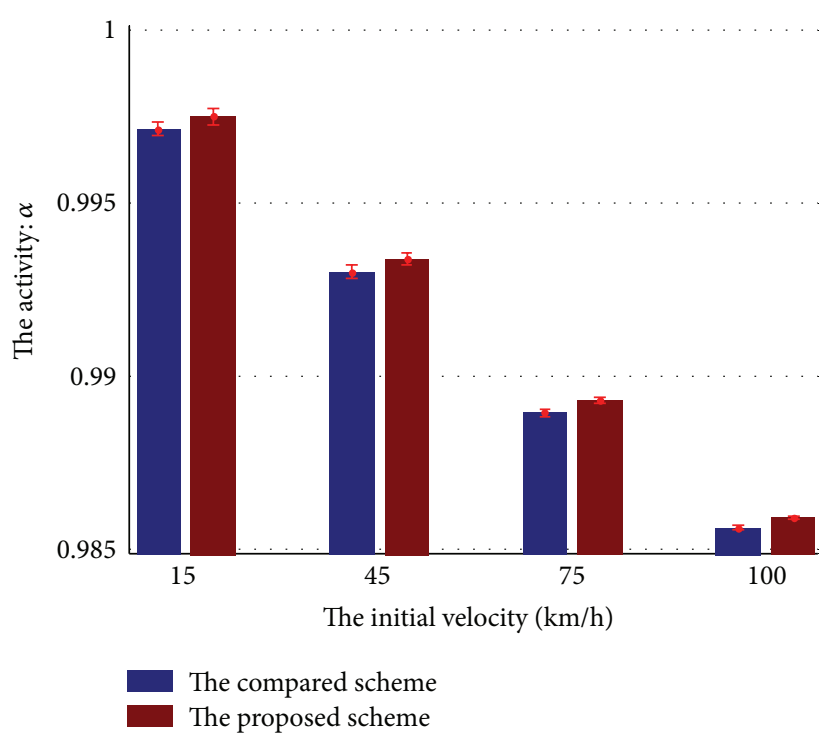

(a) The results of the activity $\alpha$

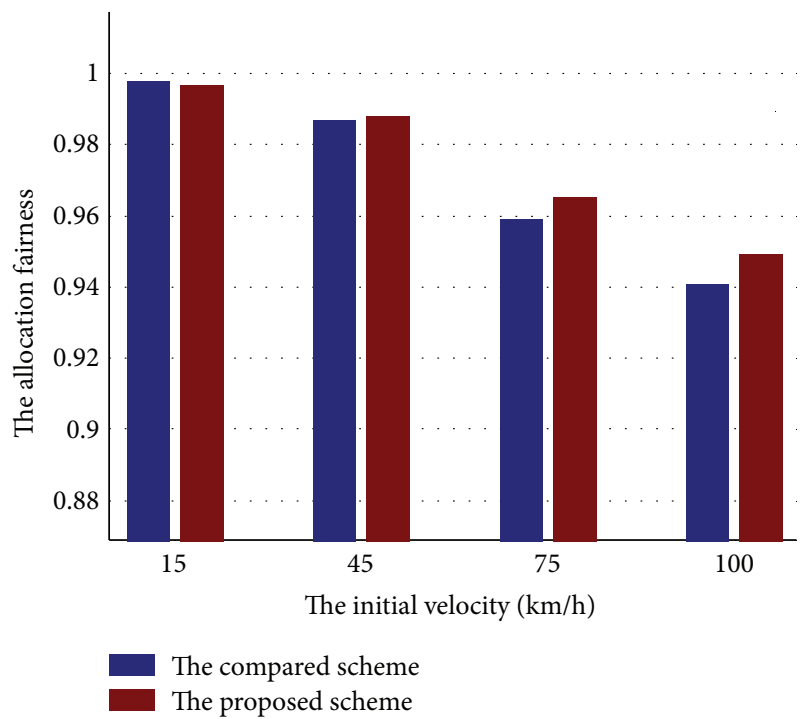

(b) The results of the fairness

FIGURE 8: The simulation results under different initial velocity.

terminals may switch their connection between the optimal and the suboptimal networks much frequently. Therefore, the compared scheme potentially increases the handover times and reduces the efficiency of network resources allocation as well as the overall level of multiple terminals' QoS satisfaction. Unlike the deterministic optimization that behaves in a greedy manner, the attractor selection mechanism searches the attractor with stochastic optimization, that is, searching the optimal or suboptimal state with some certain noises. Although the attractor selection mechanism cannot guarantee that an application is connected to the best network all the time, it drives the multiple applications to select their appropriate access wireless links in a collaborative manner and makes these applications' connection adapt to the varying wireless network conditions, meanwhile meeting their QoS requirements to some extent. The mechanism drives multiple terminals to make handover decision in the way that is similar to the coexistence and self-adaptability of multiple cells behaving in a dynamic environment. Therefore, the bio-inspired attractor selection model is able to achieve a better performance from a global perspective.

\section{Conclusions}

In this paper, we propose a bio-inspired model for making handover decision in dynamic heterogeneous wireless environment. Our scheme provides a QoS-oriented handover solution for selecting an appropriate wireless network that can well satisfy the QoS requirements of each of the individual terminal applications in the dynamic context. For supporting adaptive and automatic decision making, we have introduced the attractor selection mechanism and proposed the distributed handover decision framework based on this bio-inspired model. Then, we model the activity parameter in terms of the individual terminal QoS satisfaction by a novel utility function, treat each individual terminal as a cellular system by analogy, and use the activity as the input of the extended attractor selection model to drive the process of updating the state value of the decision matrix as well as making handover decision.

The experimental results prove that the QoS-oriented handover decision scheme induced by the bio-inspired attractor selection model achieves better adaptation to the varying heterogeneous wireless environment and has better performance in terms of guaranteeing better QoS satisfaction and ensuring better fairness of network resources allocation when compared with the traditional utility function based scheme. In the future, we will extend our scheme by taking more decision factors into account such as the user profile related and terminal related decision factors, and we will validate the proposed scheme under some more complex heterogeneous environments where more dynamic characteristics are considered.

\section{Conflict of Interests}

The authors declare that there is no conflict of interests regarding the publication of this paper.

\section{Acknowledgments}

This research is supported by the National Natural Science Foundation of China under Grant nos. 61103098 and 91118008, the National High Technology Research and Development Project (863 Project) under Grant no. 2012AA111901, the Beihang University Innovation \& Practice Funding for Graduate under Grant no. YCSJ-02-2013-01, the Foundation of Key Laboratory of Road and Traffic Engineering of the Ministry of Education in Tongji University, Bagui scholarship project, the Natural Science Foundation of Guangxi under 
Grant nos. 2011GXNSFA018154 and 2012GXNSFGA060003, the Science and Technology Foundation of Guangxi under Grant no. 10169-1, the Guangxi Scientific Research Project no. 201012MS274, the Funded Projects of Innovation Plan for Guangxi Graduate Education no. gxun-chx2013t18, the Natural Science Foundation of Guangxi under Grant no. 2013GXNSFAA019342, and the Guangxi University for Nationalities Project no. 2012QD017.

\section{References}

[1] E. Gustafsson and A. Jonsson, "Always best connected," IEEE Wireless Communications, vol. 10, no. 1, pp. 49-55, 2003.

[2] M. Zekri, B. Jouaber, and D. Zeghlache, "A review on mobility management and vertical handover solutions over heterogeneous wireless networks," Computer Communications, vol. 35, no. 17, pp. 2055-2068, 2012.

[3] X. H. Yan, Y. A. Sekercioglu, and S. Narayanan, "A survey of vertical handover decision algorithms in fourth generation heterogeneous wireless networks," Computer Networks, vol. 54, no. 11, pp. 1848-1863, 2010.

[4] F. Dressler and O. B. Akan, "A survey on bio-inspired networking," Computer Networks, vol. 54, no. 6, pp. 881-900, 2010.

[5] J.-B. Wang, M. Chen, X. Wan, and C. Wei, "Ant-colonyoptimization-based scheduling algorithm for uplink CDMA nonreal-time data," IEEE Transactions on Vehicular Technology, vol. 58, no. 1, pp. 231-241, 2009.

[6] M. Meisel, V. Pappas, and L. Zhang, "A taxonomy of biologically inspired research in computer networking," Computer Networks, vol. 54, no. 6, pp. 901-916, 2010.

[7] C. Zheng and D. Sicker, "A survey on biologically inspired algorithms for computer networking," IEEE Communications Surveys \& Tutorials, vol. 15, no. 3, pp. 1160-1191, 2013.

[8] H. Kitano, "Biological robustness," Nature Reviews Genetics, vol. 5, no. 11, pp. 826-837, 2004.

[9] H. Kitano, "Towards a theory of biological robustness," Molecular Systems Biology, vol. 3, no. 1, article 137, 2007.

[10] A. Kashiwagi, I. Urabe, K. Kaneko, and T. Yomo, "Adaptive response of a gene network to environmental changes by fitnessinduced attractor selection," PLoS ONE, vol. 1, no. 1, article e49, 2006.

[11] I. Fukuyori, Y. Nakamura, Y. Matsumoto, and H. Ishiguro, "Control method for a robot based on the adaptive attractor selection model," in Proceedings of the 4th International Conference on Autonomous Robots and Agents (ICARA '09), pp. 618623, Wellington, New Zealand, February 2009.

[12] T. Iwai, N. Wakamiya, and M. Murata, "Error-tolerant coverage control based on bio-inspired attractor selection model for wireless sensor networks," in Proceedings of the 10th IEEE International Conference on Computer and Information Technology (CIT '10), pp. 723-729, Bradford, UK, July 2010.

[13] Y. Koizumi, T. Miyamura, S. Arakawa, E. Oki, S. Kohei, and M. Murtata, "Adaptive virtual network topology control based on attractor selection," Journal of Lightwave Technology, vol. 28, no. 11, pp. 1720-1731, 2010.

[14] K. J. Leibnitz, N. Wakamiya, and M. Murata, "A bio-inspired robust routing protocol for mobile ad hoc networks," in Proceedings of the 16th International Conference on Computer Communications and Networks (ICCCN '07), pp. 321-326, Honolulu, Hawaii, USA, August 2007.
[15] S. Kajioka, N. Wakamiya, and M. Murata, "Autonomous and adaptive resource allocation among multiple nodes and multiple applications in heterogeneous wireless networks," Journal of Computer and System Sciences, vol. 78, no. 6, pp. 1673-1685, 2012.

[16] J. Hasty, J. Pradines, M. Dolnik, and J. J. Collins, "Noise-based switches and amplifiers for gene expression," Proceedings of the National Academy of Sciences of the United States of America, vol. 97, no. 5, pp. 2075-2080, 2000.

[17] M. B. Elowitz, A. J. Levine, E. D. Siggia, and P. S. Swain, "Stochastic gene expression in a single cell," Science, vol. 297, no. 5584, pp. 1183-1186, 2002.

[18] S. Sharna and M. Murshed, "Performance analysis of vertical handoff algorithms with QoS parameter differentiation," in Proceedings of the 12th IEEE International Conference on High Performance Computing and Communications (HPCC '10), pp. 623-628, Melbourne, Australia, September 2010.

[19] J.-M. Kang, J. Strassner, S.-S. Seo, and J. W.-K. Hong, "Autonomic personalized handover decisions for mobile services in heterogeneous wireless networks," Computer Networks, vol. 55, no. 7, pp. 1520-1532, 2011.

[20] T. Kang, C. Hong, Y. Kim, and S. Kim, "A context-aware handoff management for seamless connectivity in ubiquitous computing environment," in Proceedings of the International Conference on Pervasive Systems and Computing (PSC '06), pp. 128-134, Prague, Czech Republic, 2006.

[21] R. Jain, D. M. Chiu, and W. R. Hawe, "A quantitative measure of fairness and discrimination for resource allocation in shared computer system," Eastern Research Laboratory, Digital Equipment Corporation, 1984. 


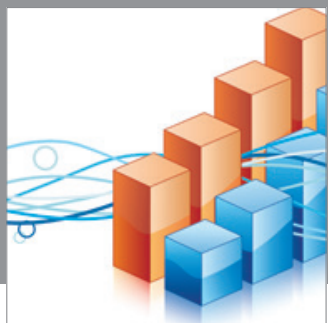

Advances in

Operations Research

mansans

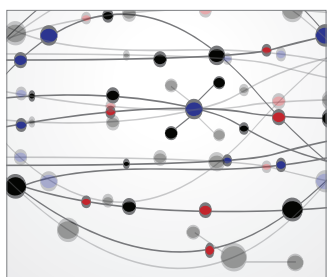

The Scientific World Journal
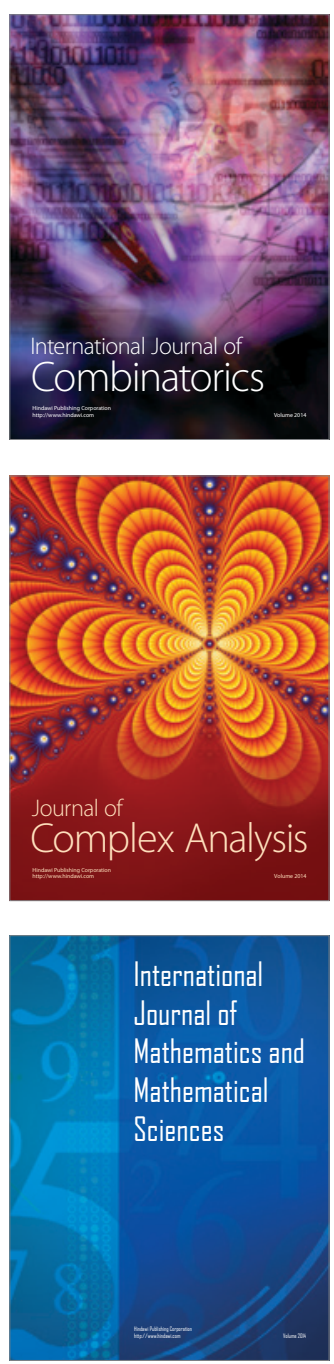
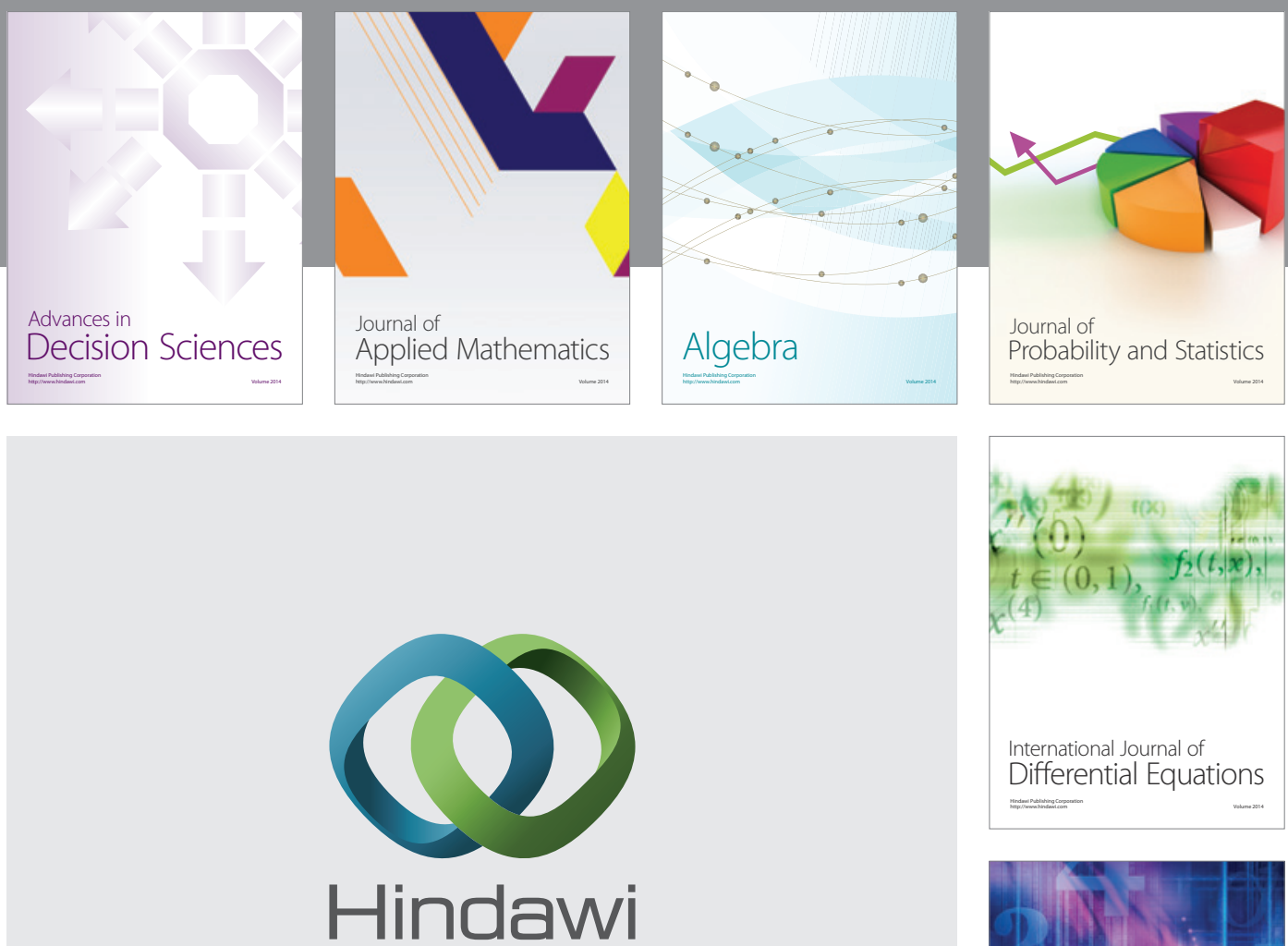

Submit your manuscripts at http://www.hindawi.com
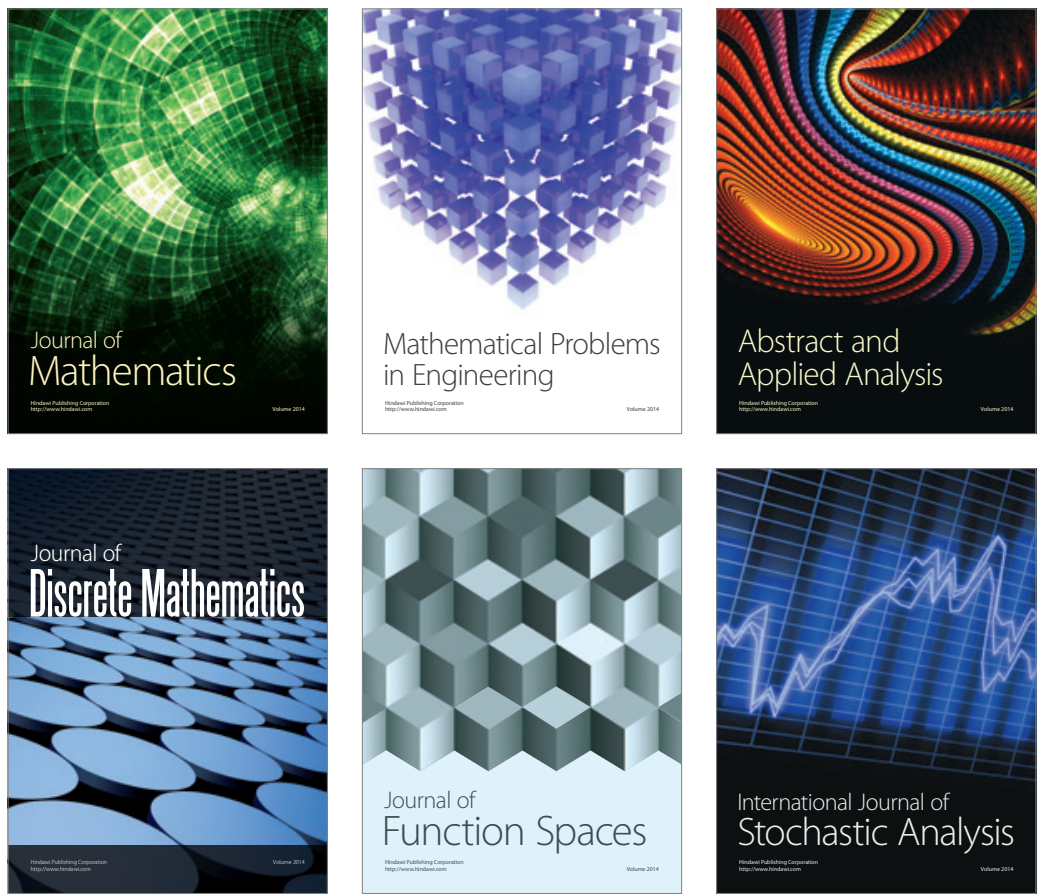

Journal of

Function Spaces

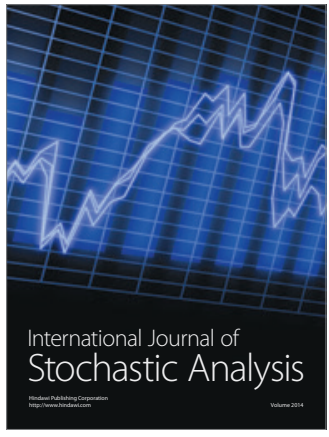

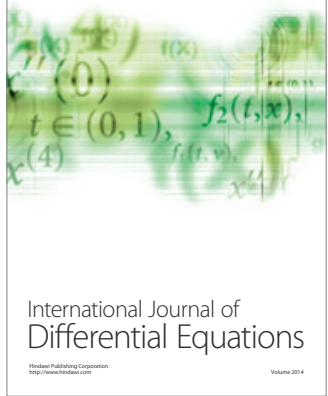
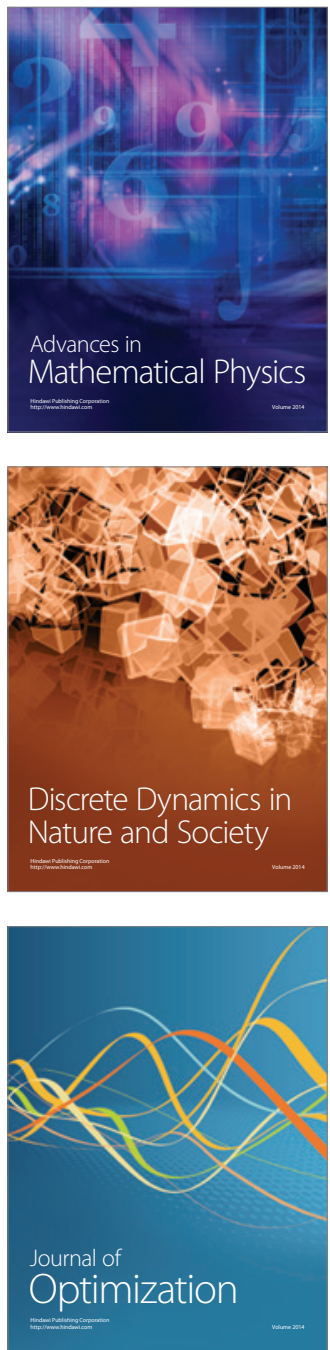